January, 1997

IFT-P/009/97

solv-int/9701013

\title{
The structures underlying soliton solutions in integrable hierarchies ${ }^{1}$
}

\author{
Luiz A. Ferreira ${ }^{2}$ \\ Instituto de Física Teórica - IFT/UNESP \\ Rua Pamplona 145 \\ 01405-900, São Paulo - SP, Brazil
}

\begin{abstract}
.
We point out that a common feature of integrable hierarchies presenting soliton solutions is the existence of some special "vacuum solutions" such that the Lax operators evaluated on them, lie in some abelian subalgebra of the associated Kac-Moody algebra. The soliton solutions are constructed out of those "vacuum solitons" by the dressing transformation procedure.
\end{abstract}

This talk is concerned with the structures responsible for the appearance of soliton solutions for a large class of non linear differential equations. In spite of the great variety of types of equations presenting soliton solutions, some basic features seem to be common to all of them. Pratically all such theories have a representation in terms of a zero curvature condition [1], and the corresponding Lax operators lie in some infinite dimensional Lie algebra, in general a Kac-Moody algebra $\hat{\mathcal{G}}$. We argue that one of the basic ingredients for the appearence of soliton solutions in such theories is the existence of "vacuum solutions" corresponding to Lax operators lying in some abelian (up to central term) subalgebra of $\hat{\mathcal{G}}$. Using the dressing transformation procedure [2] we construct the solutions in the orbit of those vacuum solutions, and conjecture that the soliton solutions correspond to some special points in those orbits. The talk is based on results obtained in collaboration with J.L. Miramontes and J. Sanchez Guillén and reported in ref. [3].

We consider non-linear integrable hierarchies of equations which can be formulated in terms of a system of first order differential

$$
\mathcal{L}_{N} \Psi=0, \quad \mathcal{L}_{N} \equiv \frac{\partial}{\partial t_{N}}-A_{N}
$$

1) Talk given at the I Latin American Symposium on High Energy Physics, I SILAFAE, Merida, Mexico, November/96

2) Partially supported by a CNPq research grant 
where the variables $t_{N}$ are the various "times" of the hierarchies, and their number may be finite or infinite. The equations of the hierarchies are then equivalent to the integrability or zero-curvature conditions of (1)

$$
\left[\mathcal{L}_{N}, \mathcal{L}_{M}\right]=0
$$

Therefore, the Lax operators are "flat connections"

$$
A_{N}=\frac{\partial \Psi}{\partial t_{N}} \Psi^{-1}
$$

The type of integrable hierarchy considered here is based on a Kac-Moody algebra $\hat{\mathcal{G}}$, furnished with an integral gradation

$$
\hat{\mathcal{G}}=\bigoplus_{i \in \mathbb{Z}} \hat{\mathcal{G}}_{i} \quad \text { and } \quad\left[\hat{\mathcal{G}}_{i}, \hat{\mathcal{G}}_{j}\right] \subseteq \hat{\mathcal{G}}_{i+j}
$$

The connections are of the form

$$
A_{N}=\sum_{i=N_{-}}^{N_{+}} A_{N, i}, \quad \text { where } \quad A_{N, i} \in \hat{\mathcal{G}}_{i}
$$

where $N_{-}$and $N_{+}$are non-positive and non-negative integers, respectively.

We assume that the hierarchy posssesses at least one vacuum solution such that the connections evaluated on such solution has the form

$$
A_{N}^{(\mathrm{vac})}=\sum_{i=N_{-}}^{N_{+}} c_{N}^{i} b_{i}+f_{N}(t) C \equiv \varepsilon_{N}+f_{N}(t) C .
$$

where $C$ is the central element of $\hat{\mathcal{G}}$ and $b_{i} \in \hat{\mathcal{G}}_{i}$ are generators of a subalgebra of $\hat{\mathcal{G}}$ satisfying

$$
\left[b_{j}, b_{k}\right]=j \beta_{j} C \delta_{j+k, 0}
$$

with $\beta_{j}$ being some complex numbers $\left(\beta_{-j}=\beta_{j}\right), c_{N}^{i}$ are constants, and $f_{N}(t)$ are functions ot the times $t_{N}$. ${ }^{3}$ These vacuum potentials correspond to the solution of the associated linear problem (1)-(3), given by the group element

$$
\Psi^{(\mathrm{vac})}=\exp \left(\sum_{N} \varepsilon_{N} t_{N}+\gamma(t) C\right)
$$

where the numeric function $\gamma(t)$ is a solution of the equations

$$
\frac{\partial \gamma(t)}{\partial t_{N}}=f_{N}(t)+\frac{1}{2} \sum_{M, i} i \beta_{i} c_{N}^{i} c_{M}^{-i} t_{M} .
$$

3) As a consequence of (2), those functions have to satisfy $\frac{\partial f_{N}(t)}{\partial t_{M}}-\frac{\partial f_{M}(t)}{\partial t_{N}}=\sum_{i} i \beta_{i} c_{M}^{i} c_{N}^{-i}$ 
We now consider the dressing tranformations which map known solutions of the hierarchy into new solutions [2]. Denote by $\hat{G}_{-}, \hat{G}_{+}$, and $\hat{G}_{0}$ the subgroups of the Kac-Moody group $\hat{G}$ formed by exponentiating the subalgebras $\hat{\mathcal{G}}_{<0} \equiv$ $\bigoplus_{i<0} \hat{\mathcal{G}}_{i}, \hat{\mathcal{G}}_{>0} \equiv \bigoplus_{i>0} \hat{\mathcal{G}}_{i}$, and $\hat{\mathcal{G}}_{0}$, respectively. According to Wilson [4], the dressing transformations can be described in the following way. Consider a solution $\Psi$ of the linear problem (1), and let $\rho=\rho_{-} \rho_{0} \rho_{+}$be a constant element in the "big cell" of $\hat{G}$, i.e., in the subset $\hat{G}_{-} \hat{G}_{0} \hat{G}_{+}$of $\hat{G}$, such that

$$
\Psi \rho \Psi^{-1}=\left(\Psi \rho \Psi^{-1}\right)_{<0}\left(\Psi \rho \Psi^{-1}\right)_{0}\left(\Psi \rho \Psi^{-1}\right)_{>0} .
$$

Notice that these conditions are equivalent to say that both $\rho$ and $\Psi \rho \Psi^{-1}$ admit a generalized Gauss decomposition with respect to the gradation (4). Define

$$
\begin{aligned}
\Psi^{\rho} & =\Theta_{-}^{(0)}\left[\left(\Psi \rho \Psi^{-1}\right)_{<0}\right]^{-1} \Psi \rho \equiv \Theta_{-} \Psi \rho \\
& =\Theta_{+}^{(0)}\left(\Psi \rho \Psi^{-1}\right)_{>0} \Psi \equiv \Theta_{+} \Psi
\end{aligned}
$$

where

$$
\Theta_{-}^{(0)}{ }^{-1} \Theta_{+}^{(0)}=\left(\Psi \rho \Psi^{-1}\right)_{0}
$$

Then, $\Psi^{\rho}$ is another solution of the linear problem. Indeed, one can check that by exploring the gradation of $\hat{\mathcal{G}}$ and the fact that the dressing transformation is written in two ways (11), that the transformed connection lie in the same subspace of $\hat{\mathcal{G}}$ as the original one, i.e.

$$
A_{N}^{\rho}=\frac{\partial \Psi^{\rho}}{\partial t_{N}}\left(\Psi^{\rho}\right)^{-1} \in \bigoplus_{i=N_{-}}^{N_{+}} \hat{\mathcal{G}}_{i},
$$

We now consider the orbit of the vacuum solution (8) under the group of dressing transformations. For each constant group element $\rho$ one gets a new solution, out of the vacuum solution, by the transformation $\Psi^{(\mathrm{vac})} \mapsto \Psi^{\rho}=\Theta_{-} \Psi^{(\mathrm{vac})} \rho=\Theta_{+} \Psi^{(\mathrm{vac})}$. The the vacuum connection $A_{N}^{(\mathrm{vac})}$ becomes

$$
\begin{aligned}
A_{N}^{\rho}-f_{N}(t) c & =\Theta_{-} \varepsilon_{N} \Theta_{-}^{-1}+\partial_{N} \Theta_{-} \Theta_{-}^{-1} \in \bigoplus_{i \leq N_{+}} \hat{\mathcal{G}}_{i} \\
& =\Theta_{+} \varepsilon_{N} \Theta_{+}{ }^{-1}+\partial_{N} \Theta_{+} \Theta_{+}{ }^{-1} \in \bigoplus_{i \geq N_{-}} \hat{\mathcal{G}}_{i},
\end{aligned}
$$

The components $A_{N, i}$ in (5), of the connection are functionals of the fields of the hierarchy. Then one can consider (14) as a local change of variables, and use it to relate the parameters of the group elements $\Theta_{+}$and $\Theta_{-}$, to the fields of the hierarchy. In fact, one can choose a suitable set of parameters to write the fields in terms of them. The value of that particular set of parameters evaluated on the solution can be obtained by considering matrix elements of the form 


$$
\left\langle\mu\left|\Theta_{-}^{-1} \Theta_{+}\right| \mu^{\prime}\right\rangle=\left\langle\mu\left|e^{\sum_{N} \varepsilon_{N} t_{N}} \rho e^{-\sum_{N} \varepsilon_{N} t_{N}}\right| \mu^{\prime}\right\rangle,
$$

where $|\mu\rangle$ and $\left|\mu^{\prime}\right\rangle$ are vectors in a given representation of $\hat{\mathcal{G}}$. The appropriate set of vectors is specified by the condition that all the required components of $\Theta_{-}$ and $\Theta_{+}$, used to parametrize the fields, can be expressed in terms of the resulting matrix elements. It turns out [3] that the required matrix elements, considered as functions of the group element $\rho$, constitute the generalization of the Hirota's tau-functions for these hierarchies [5]. Moreover, Eq. (15) is the analogue of the, so called, solitonic specialization of the Leznov-Saveliev solution proposed in [6-9] for the affine (abelian and non-abelian) Toda theories.

Consider now the common eigenvectors of the adjoint action of the $\varepsilon_{N}$ 's that specify the vacuum solution (6). Then, the important class of multi-soliton solutions is conjectured to correspond to group elements $\rho$ which are the product of exponentials of eigenvectors

$$
\rho=e^{F_{1}} e^{F_{2}} \ldots e^{F_{n}}, \quad\left[\varepsilon_{N}, F_{k}\right]=\omega_{N}^{(k)} F_{k}, \quad k=1,2, \ldots n .
$$

In this case, the dependence of the solution upon the times $t_{N}$ can be made quite explicit

$$
\left\langle\mu\left|\Theta_{-}^{-1} \Theta_{+}\right| \mu^{\prime}\right\rangle=\left\langle\mu\left|\prod_{k=1}^{n} \exp \left(e^{\sum_{N} \omega_{N}^{(k)} t_{N}} F_{k}\right)\right| \mu^{\prime}\right\rangle .
$$

We emphasize that not all solutions of the type (17) are soliton solutions, but we conjecture that the soliton and multi-soliton solutions are among them. The conjecture that multi-soliton solutions are associated with group elements of the form (16) naturally follows from the well known properties of the multi-soliton solutions of affine Toda equations and of hierarchies of the $\mathrm{KdV}$ type, and, in the sine-Gordon theory, it has been explicitly checked in ref. [10]. Actually, in all these cases, the multi-soliton solutions are obtained in terms of representations of the "vertex operator" type where the corresponding eigenvectors are nilpotent. Then, for each eigenvector $F_{k}$ there exists a positive integer number $m_{k}$ such that $\left(F_{k}\right)^{m} \neq 0$ only if $m \leq m_{k}$. This remarkable property simplifies the form of (17 ) because it implies that $e^{F_{k}}=1+F_{k}+\cdots+\left(F_{k}\right)^{m_{k}} / m_{k}$ !, which provides a group-theoretical justification of Hirota's method [5].

An interesting feature of the dressing transformations method is the possibility of relating the solutions of different integrable equations. Consider two different integrable hierarchies whose vacuum solutions are compatible, in the sense that the corresponding vacuum Lax operators commute. Then, one can consider the original integrable equations as the restriction of a larger hierarchy of equations. Consequently, the solutions obtained through the group of dressing transformations can also be understood in terms of the solutions of the larger hierarchy, which implies certain relations among them. (see section 4 of [8] for more details).

The developments described here lead to a quite general definition of tau functions for such hierachies, in terms of integrable highest weight representations of the associated Kac-Moody algebra [3]. 


\section{Acknowledgements}

The author is very grateful to the organizers of I SILAFAE and VII EMPC, and to CLAF (Centro Latino Americano de Física) for the finantial support.

\section{REFERENCES}

1. V.E. Zakharov, A.B. Shabat, Functional Analysis and its Application 13 (1979) 166.

2. E. Date, M. Jimbo, M. Kashiwara, T. Miwa, Proc. Japan. Acad. 57 (1981) 3806; Physica 4 (1982) 343; Publ. RIMS Kyoto University 18, (1982) 1077; M. SemenovTian-Shansky, Functional Analysis and its Application 17 (1983) 259; Publ. RIMS Kyoto Univ. 21 (1985) 1237.

3. L.A. Ferreira, J.L. Miramontes and J. Sánchez Guillén, Journal of Mathematical Physics 38 \# 2, (1997), hep-th/9606066.

4. G. Wilson, Phil. Trans. R. Soc. Lond. A 315 (1985) 383; Habillage et fonctions $\tau$ C. R. Acad. Sc. Paris 299 (I) (1984) 587; The $\tau$-Functions of the gAKNS Equations, in "Verdier memorial conference on integrable systems" (O. Babelon, P. Cartier, and Y. Kosmann-Schwarzbach, eds.), Birkhauser (1993) 131-145.

5. R. Hirota, Direct methods in soliton theory, in "Soliton" (R.K. Bullough and P.S. Caudrey, eds.), (1980) 157.; J. Phys. Soc. Japan 33 (1972) 1459.

6. D. Olive, N. Turok and J.W.R. Underwood, Nucl. Phys. B401 (1993) 663-697.; Nucl. Phys. B409 (1993) 509-546, hep-th/9305160.

7. D. Olive, M.V. Saveliev and J.W.R. Underwood, Phys. Lett. B311 (1993) 117-122, hep-th/9212123.

8. L.A. Ferreira, J.L. Miramontes and J. Sánchez Guillén, Nucl. Phys. 449 (1995) 631679, hep-th/9412127.

9. L.A. Ferreira, J-L. Gervais, J. Sánchez Guillén and M.V. Saveliev, Affine Toda Systems Coupled to Matter Fields, Nucl. Phys. B470 (1996) 236, hep-th/9512105.

10. O. Babelon and D. Bernard, Int. J. Mod. Phys. A8 (1993) 507-543, hep-th/9206002. 